\title{
Supercooling of rapidly expanding quark-gluon plasma
}

\author{
E.E. Zabrodin ${ }^{a, b}$, L.V. Bravina ${ }^{a, b, 1}$, L.P. Csernai ${ }^{c}$, H. Stöcker ${ }^{a}$ \\ and W. Greiner ${ }^{a}$ \\ ${ }^{a}$ Institute for Theoretical Physics, University of Frankfurt, \\ Robert-Mayer-Str. 8-10, D-60054 Frankfurt, Germany \\ ${ }^{b}$ Institute for Nuclear Physics, Moscow State University, 119899 Moscow, Russia \\ ${ }^{c}$ Department of Physics, University of Bergen, Allégaten 55, 5007 Bergen, Norway \\ 1 Alexander von Humboldt Fellow
}

\begin{abstract}
We reexamine the scenario of homogeneous nucleation of the quark-gluon plasma produced in ultra-relativistic heavy ion collisions. A generalization of the standard nucleation theory to rapidly expanding system is proposed. The nucleation rate is derived via the new scaling parameter $\lambda_{Z}$. It is shown that the size distribution of hadronic clusters plays an important role in the dynamics of the phase transition. The longitudinally expanding system is supercooled to about $3-6 \%$, then it is reheated, and the hadronization is completed within $6-10 \mathrm{fm} / c$, i.e. $5-10$ times faster than it was estimated earlier, in a strongly nonequilibrium way.
\end{abstract}

Key words: QGP-hadrons phase transition; Homogeneous nucleation; Cluster distribution; Bjorken model.

PACS: 12.38.Mh; 12.39.Ba; 25.75.-q; 64.60.Qb 
Homogeneous nucleation of quark-gluon plasma (QGP) produced in ultrarelativistic heavy ion collisions has been subject of interest during the last few years. Obviously, the plasma must hadronize, but the mechanism of hadronization still remains an open question. In case of a second order phase transition, percolation model calculations are useful, also for strong supercooling of the plasma during a first order phase transition, provided the expansion is rapid and the amount of the plasma converted into hadronic matter is not sufficiently large to reheat the growing volume.

Results of simulations $[1,2]$ show that the supercooling of the system, if quenched rapidly into the metastable or even unstable region, may be as large as $20 \%$. Then it is questionable whether the homogeneous nucleation scheme is appropriate for the description of the hadronization process. Other processes which may cause a rapid hadronization of QGP have been studied recently [3,4].

In the present paper we propose a generalization of the standard nucleation theory to rapidly expanding systems. If the size distribution of hadronic bubbles is taken into account, the total rate of the plasma conversion turns out to be high enough to prevent the system from strong supercooling. The last circumstance makes relevant the application of the homogeneous nucleation scenario.

The paper is organized as follows: after a sketch of the nucleation theory the dynamics of the plasma-hadrons phase transition in the Bjorken hydrodynamic model [5] is discussed. Finally, conclusions will be drawn.

The starting point of the homogeneous nucleation theory is the assumption of formation of nucleating clusters within the initially homogeneous metastable state. The rate of conversion of the metastable state into a thermodynamically stable phase is called nucleation rate. According to Langer [6], who has extended the classical nucleation theory (see, e.g. [7] and references herein) to field theories, the rate of the relaxation of a metastable state is given by

$$
I=\frac{\kappa}{2 \pi} \Omega_{0} \exp \left(-\beta \Delta F_{*}\right)
$$

where $\kappa$ and $\Omega_{0}$ is a dynamical prefactor and a statistical prefactor, respectively, $\Delta F_{*}$ is the excess free energy of the cluster of critical size, $\beta \equiv\left(k_{B} T\right)^{-1}$, $k_{B}$ is the Boltzmann constant, and $T$ is the temperature of the system.

Let us consider a classical system with $N$ degrees of freedom described by a set of $N$ collective coordinates $\xi_{i}, i=1, \ldots, N$. The energy function $E(\{\xi\})$ of the system should have two locally stable states separated by the energy barrier. The point with minimal energy on the barrier $\left\{\xi^{S}\right\}$ is the so-called saddle point. 
The statistical prefactor $\Omega_{0}$ is a measure of the available phase space volume of the saddle point region. If the fluctuation corrections to the (mean field) excess free energy of the cluster are absorbed into $\Delta F$, the statistical prefactor [6] can be written as

$$
\Omega_{0}=\mathcal{V}\left(\frac{2 \pi}{\beta\left|\lambda_{1}\right|}\right)^{1 / 2}
$$

where $\mathcal{V}=V\left(\frac{8 \pi \sigma}{3\left|\lambda_{1}\right|}\right)^{3 / 2}$ is the available volume of the saddle point subspace, $V$ is the volume of the system, $\sigma$ is the surface tension, and $\lambda_{1}$ is the only negative eigenvalue of the matrix $\frac{\partial^{2} E}{\partial \xi_{i} \partial \xi_{j}}$ defined at the saddle point $\left\{\xi^{S}\right\}$.

The dynamical prefactor $\kappa$ determines the growth rate of the critical cluster at the saddle point. For a relativistic system of particles, where the thermal conductivity is absent because of the absence of the rest frame defined by the baryon net charge, the dynamical prefactor has been calculated [8] to be

$$
\kappa=\frac{4 \sigma\left(\zeta+\frac{4}{3} \eta\right)}{(\Delta \omega)^{2} R_{*}^{3}} .
$$

Here $\zeta$ and $\eta$ is the shear and bulk viscosity, $\Delta \omega$ is the difference in the enthalpy densities of the quark-gluon phase and hadronic phase, and $R_{*}$ is the radius of the critical cluster.

We will explore a model QGP, produced in the collision of ultrarelativistic heavy ions, and subsequently rapidly quenched into the metastable region below the critical temperature. Then the first order phase transition should occur. It will be triggered by the creation of hadronic clusters as a result of the thermodynamic fluctuations. The Fisher droplet model [9] yields the change in the free energy of the system due to the formation of a spherical cluster of radius $R$

$$
\Delta F(R)=-\frac{4 \pi}{3} R^{3} \Delta p+4 \pi R^{2} \sigma+3 \tau_{F} \beta^{-1} \ln \frac{R}{r_{0}} .
$$

Here $\Delta p=p_{h}-p_{q q p}$ is the difference of the pressures inside and outside the cluster, $\tau_{F}=2.2$ is the critical exponent, and $r_{0}$ is the radius of the smallest cluster formed in the system. The maximum of the $\Delta F(R)$ will be reached at the critical radius

$$
R_{*}=\left(a_{1}+\sqrt{a_{2}^{3}+a_{1}^{2}}\right)^{1 / 3}+\left(a_{1}-\sqrt{a_{2}^{3}+a_{1}^{2}}\right)^{1 / 3}+a_{3},
$$


containing $a_{1}=\frac{9 \tau_{F} a_{3}}{4 \alpha^{2}}+a_{3}^{3}, \quad a_{2}=-a_{3}^{2}, \quad a_{3}=\frac{2 \sigma}{3 \Delta p}, \quad \alpha=\sqrt{4 \pi \sigma \beta}$.

Clusters whose radii are smaller than $R_{*}$ are shrinking, clusters whose radii are larger than $R_{*}$ are growing, and clusters of critical size are in metastable equilibrium. It is convenient to parametrize the excess free energy of a cluster by the similarity number $\lambda_{Z}=R_{*} \sqrt{4 \pi \sigma \beta}$ [10] and reduced radius $r=R / R_{*}$

$$
\beta \Delta F=-\left(\tau_{F}+\frac{2}{3} \lambda_{Z}^{2}\right) r^{3}+\lambda_{Z}^{2} r^{2}+3 \tau_{F} \ln \frac{r R_{*}}{r_{0}}
$$

Then for the only negative eigenvalue $\lambda_{1}$, associated with the instability of the critical cluster against growth or shrinking, we have

$$
\lambda_{1}=-\left(9 \tau_{F}+2 \lambda_{Z}^{2}\right) \beta^{-1} .
$$

Substitution of this result in Eq. (2) gives finally for the statistical prefactor

$$
\Omega_{0}=\frac{4 \sqrt{\pi}}{3 \sqrt{3}}\left(\frac{\lambda_{Z}}{R_{*}}\right)^{3} \frac{V}{\left(9 \tau_{F}+2 \lambda_{Z}^{2}\right)^{2}} .
$$

The result for the total nucleation rate $I$ reads

$$
I=V \frac{8}{3 \sqrt{3 \pi}}\left(\frac{\lambda_{Z}}{R_{*}^{2}}\right)^{3} \frac{\sigma\left(\zeta+\frac{4}{3} \eta\right)}{(\Delta \omega)^{2}\left(9 \tau_{F}+2 \lambda_{Z}^{2}\right)^{2}} \exp \left(-\beta \Delta F_{*}\right),
$$

where $\Delta F_{*}=-\frac{\tau_{F}}{\beta}\left(1-3 \ln \frac{R_{*}}{r_{0}}\right)+\frac{\lambda_{Z}^{2}}{3 \beta}$ is the free energy of the critical cluster. The formula (9) is similar to, but also different from the earliar expression [8]. Near the critical temperature this rate drops since the critical radius $R_{*}$ increases to infinity. Then the nucleation rate rises quickly when the temperature falls, and the first order phase transition begins.

From the definition of the nucleation rate, this is just a number of viable clusters in volume $V$ passed through the critical region per unit of time. If the total volume of the hadronic phase is not large enough to maintain the temperature of the expanding system at a constant level due to the release of the latent heat, the temperature will continue to decrease.

On the other hand, the critical radius drops very quickly with decreasing temperature. Therefore, the subcritical clusters which started to dissolve (Fig. 1, upper panel) suddenly become of supercritical size with respect to the new critical radius $R_{c}^{\prime}$ of the system (Fig. 1, lower panel). The general picture of the conversion of QGP into hadronic clusters will be rather complex. Clusters large 
enough will grow, the smallest ones will continue to shrink, while the clusters of medium size will grow or shrink depending on temperature variations and current critical radii in the system.

It is worth noting that the model presented above is valid for the hadronization of thermalized QGP produced in a large volume (of about $500 \mathrm{fm}^{3}$ or more). For smaller systems, the finite size effects [11] lead to the shift of the critical temperature and rounding of the phase transition, but the discussion of these problems lies out of scope of the present paper.

At given temperature $T$, the size distribution of clusters in the droplet model with the curvature energy is found to be [12]

$$
\begin{aligned}
f(R) & =\frac{I}{\sqrt{2 \pi\left(9 \tau_{F}+2 \lambda_{Z}^{2}\right)}} \exp \left[\frac{9 \tau_{F}+2 \lambda_{Z}^{2}}{2}(r-1)^{2}\right] \\
& \times \int_{r}^{\infty} a\left[3 \tau_{F}\left(a^{2}+a+1\right)+2 \lambda_{Z}^{2} a^{2}\right] \exp \left[-\frac{9 \tau_{F}+2 \lambda_{Z}^{2}}{2}(a-1)^{2}\right] d a
\end{aligned}
$$

Hadronic matter in our calculations is represented by a discrete spectrum of spherical bubbles starting from $r_{0}=1 \mathrm{fm}$.

Within the framework of the bag model the equations of state for two-flavour QGP and for hadronic gas are

$$
\begin{aligned}
p_{\text {qgp }} & =\frac{37}{90} \pi^{2} T^{4}-B \\
p_{h} & =\frac{1}{30} \pi^{2} T^{4} .
\end{aligned}
$$

With the bag constant $B^{1 / 4}=235 \mathrm{MeV}$ this gives us the critical temperature $T_{c}=169 \mathrm{MeV}$.

The Bjorken model of longitudinal expansion [5] yields for the evolution of the energy density $e$

$$
\frac{d e}{d \tau}=-\frac{e+p}{\tau}
$$

The energy density of the whole system, as well as the enthalpy, is a linear combination of the energy density of the plasma $e_{q g p}$ in the part of volume occupied by plasma, $\nu_{q g p}=V_{q g p} / V_{t o t}$, and the energy density of hadronic phase $e_{h}$ in the rest of the volume, $\nu_{h}=1-\nu_{q g p}$,

$$
e=e_{q g p} \nu_{q g p}+\left(1-\nu_{q g p}\right) e_{h}
$$


To determine the viscous term in Eq. (3), note that the bulk viscosity is much smaller as compared with the shear viscosity, which has been derived in leading logarithmic order of QCD [13] for a QGP with two flavours as

$$
\eta=\frac{1.29 T^{3}}{\alpha_{S}^{2} \ln \left(1 / \alpha_{S}\right)} .
$$

Here $\alpha_{S}=0.23$ is the strong coupling constant.

The last important parameter for our analysis is the value of the surface tension $\sigma$. Recent lattice QCD calculations [14,15] predict $0.01<\sigma / T_{c}^{3}<0.1$, i.e. $1.25<\sigma<12.5 \mathrm{MeV} / \mathrm{fm}^{2}$ for the given $T_{c}$. We will use $\sigma=2$ and 5 $\mathrm{MeV} / \mathrm{fm}^{2}$ in our simulations.

Following $[1,2]$, our scenario of longitudinal expansion presumes that the system reaches the critical temperature at the time $\tau_{c r}=8 \tau_{\text {init }}=3 \mathrm{fm} / \mathrm{c}$. From Fig. 2 it is apparent that the large amount of latent heat, released during the conversion process, is sufficient to prevent the expanding system from the supercooling below $3-6 \%$.

To investigate the effect of the dissipative processes on the course of the phase transition, Fig. 2 shows also results of calculations, in which Eq. (13) has been replaced [16] by

$$
\frac{d e}{d \tau}=-\frac{e+p}{\tau}+\frac{\zeta+\frac{4}{3} \eta}{\tau^{2}}
$$

One can see that the supercooling of a viscous plasma is weaker than that of an ideal QGP. This circumstance delays the homogeneous nucleation by only about $2-3 \mathrm{fm} / c$. Creation of hadronic bubbles is the main mechanism of the QGP conversion at the earlier stage of the transition. Apart from the first $\mathrm{fm} / c$ of cooling the mixed system is reheated continuously, and the temperature approaches the critical one.

This occurs up to $\approx 6 \mathrm{fm} / c$, then the behaviour of the system changes drastically: diffusion growth of the "old" bubbles (Fig. 3, upper panels) dominates the creation of new bubbles, which is practically turned off. When $T$ is close to $T_{c}$, the growth process stops, but it starts again immediately after a small extra-cooling of the system. This is a highly unstable region. Even an insignificant rise of the temperature is sufficient for the system to hit the critical point, then the homogeneous nucleation scheme is no longer relevant. Therefore, the spinodal decomposition or the percolation scenario may be appropriate to describe the strongly non-equilibrium hadronization of the rest of the QGP, which constitutes about $30-35 \%$ of the total volume. 
The size distribution of the hadronic bubbles at different stages of the phase transition is shown in lower panels of Fig. 3. We see that the initially broad plateau of the distribution function becomes narrower with the rise of the radius due to the increase of the number of bubbles per unit of radial interval. The distribution functions reach their maximum values with a radius of about $3 \leq r \leq 4.5 \mathrm{fm}$. At the very beginning of the nucleation almost all bubbles enlarge their volumes. Then the bubbles of $r \leq 1.5 \mathrm{fm}$ dissolve because their radii are smaller than the critical one. In contrast, the bubbles of $r \geq 4 \mathrm{fm}$ will stop to grow only if the temperature of the system will be very close to the critical temperature. Bubbles of size $1.5 \leq r \leq 4 \mathrm{fm}$ are either growing or shrinking due to the variations of the critical radius in the system. This leads to the appearance of the irregularities in the spectrum of small and intermediate-size bubbles (Fig. 3, right lower panel).

As a consequence, there will be no unique source of pion emission at the freezeout. The individual pairs of pions are coming from sources of $r \approx 1.5-2 \mathrm{fm}$, while the major part of pions should come from relatively large sources of $3 \leq r \leq 4.5 \mathrm{fm}$. This can be checked by the analysis of the particle correlation data and by the rapidity distributions, if the hadronic clusters will be well separated along the expansion axis; this problem has to be investigated.

In conclusion, we study two-flavour QGP undergoing a first order phase transition during the longitudinal expansion. The nucleation rate is derived via the new scaling parameter $\lambda_{Z}$. It is shown that the distribution of hadronic clusters in size plays an essential role in the dynamics of plasma hadronization near the critical temperature: there is a significant variation of the value of critical cluster in the system. The supercooling of QGP is found to be relatively moderate, $3-6 \%$ only, therefore the application of the homogeneous nucleation scenario seems to be quite reasonable.

The completion time of the transition varies from 6 to $10 \mathrm{fm} / c$. It is strongly dependent on the absolute value of the interfacial energy. The weaker the surface tension $\sigma$ of the interface is, the faster the hadronization will be, and vice versa. Since recent lattice QCD results [15] favour lower values of $\sigma$, it is likely that the QGP hadronizes within first eight $\mathrm{fm} / \mathrm{c}$ 's, i.e. very close to the currant estimations of the freeze-out time scale based on HBT data and microscopic models. Thus the completion time of the homogeneous nucleation stage is about 5-10 times shorter than those of the standard nucleation scheme obtained before $[1,2]$.

The size distribution of the hadronic clusters indicates that most of the pions are emitted from sources of about $3 \leq r \leq 4.5 \mathrm{fm}$. This signal may be investigated experimentally by means of the particle interferometry and, probably, by the rapidity spectra of secondaries on an event-by-event basis. 
Acknowledgements. We thank M.I. Gorenstein for useful discussions. L.B. and E.Z. are grateful to the Institute for Theoretical Physics, University of Frankfurt for the warm and kind hospitality. L.B. acknowledges support of the Alexander-von-Humboldt Foundation. 


\section{References}

[1] L.P. Csernai, J.I. Kapusta, Phys. Rev. Lett. 69 (1992) 737.

[2] L.P. Csernai, J.I. Kapusta, Gy. Kluge, E.E. Zabrodin, Z. Phys. C 58 (1993) 453.

[3] T. Csörgö, L.P. Csernai, Phys. Lett. B 333 (1994) 494.

[4] L.P. Csernai, I. N. Mishustin, Phys. Rev. Lett. 74 (1995) 5005.

[5] J.D. Bjorken, Phys. Rev. D 27 (1983) 140.

[6] J.S. Langer, Ann. Phys. (N.Y.) 41 (1967) 108; 54 (1969) 258.

[7] J. Frenkel, Kinetic Theory of Liquids (Clarendon, Oxford, 1946), Ch. 7.

[8] L.P. Csernai, J.I. Kapusta, Phys. Rev. D 46 (1992) 1379.

[9] M.E. Fisher, Physics (NY) 3 (1967) 255.

[10] L.V. Bravina, E.E. Zabrodin, Phys. Lett. A 202 (1995) 61.

[11] C. Spieles, H. Stöcker, C. Greiner, hep-ph/9708280.

[12] L.V. Bravina, E.E. Zabrodin, Phys. Rev. C 54 (1996) 2493.

[13] H. Heiselberg, Phys. Rev. D 49 (1994) 4739.

[14] Y. Iwasaki et al., Phys. Rev. D 49 (1994) 3540.

[15] F. Karsch et al., Nucl. Phys. B (Proc. Suppl.) 53 (1997) 413.

[16] P. Danielewicz, M. Gyulassy, Phys. Rev. D 31 (1985) 53. 

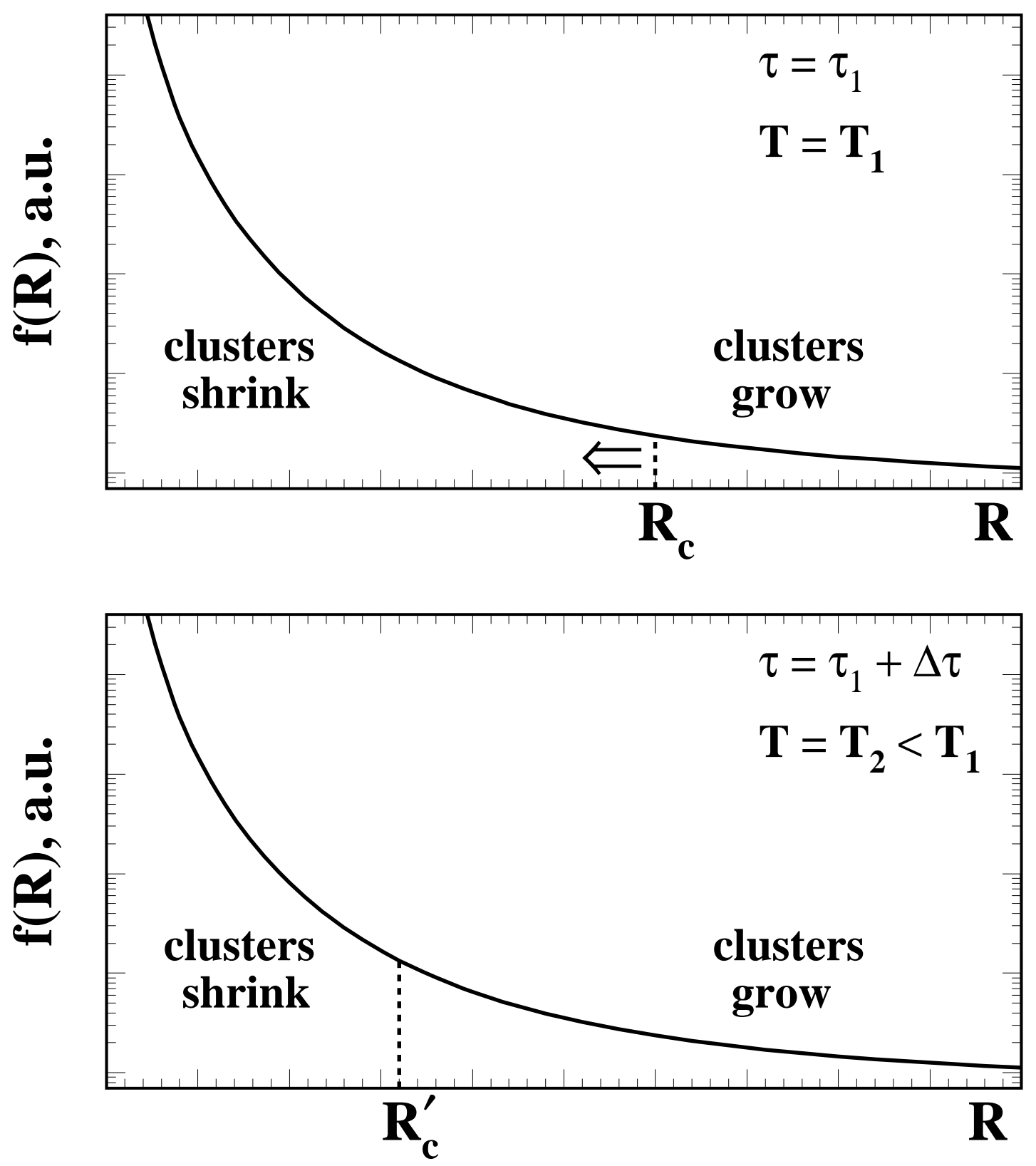

Fig. 1. Distribution of clusters as a function of their radius $R$. Due to the rapid falloff of the critical radius with temperature, the clusters of subcritical sizes (upper panel) formed at the moment $\tau_{1}$ become supercritically sized (lower panel) at $\tau_{1}+\Delta \tau$. Consequently, the amount of matter converted into hadrons increases. 


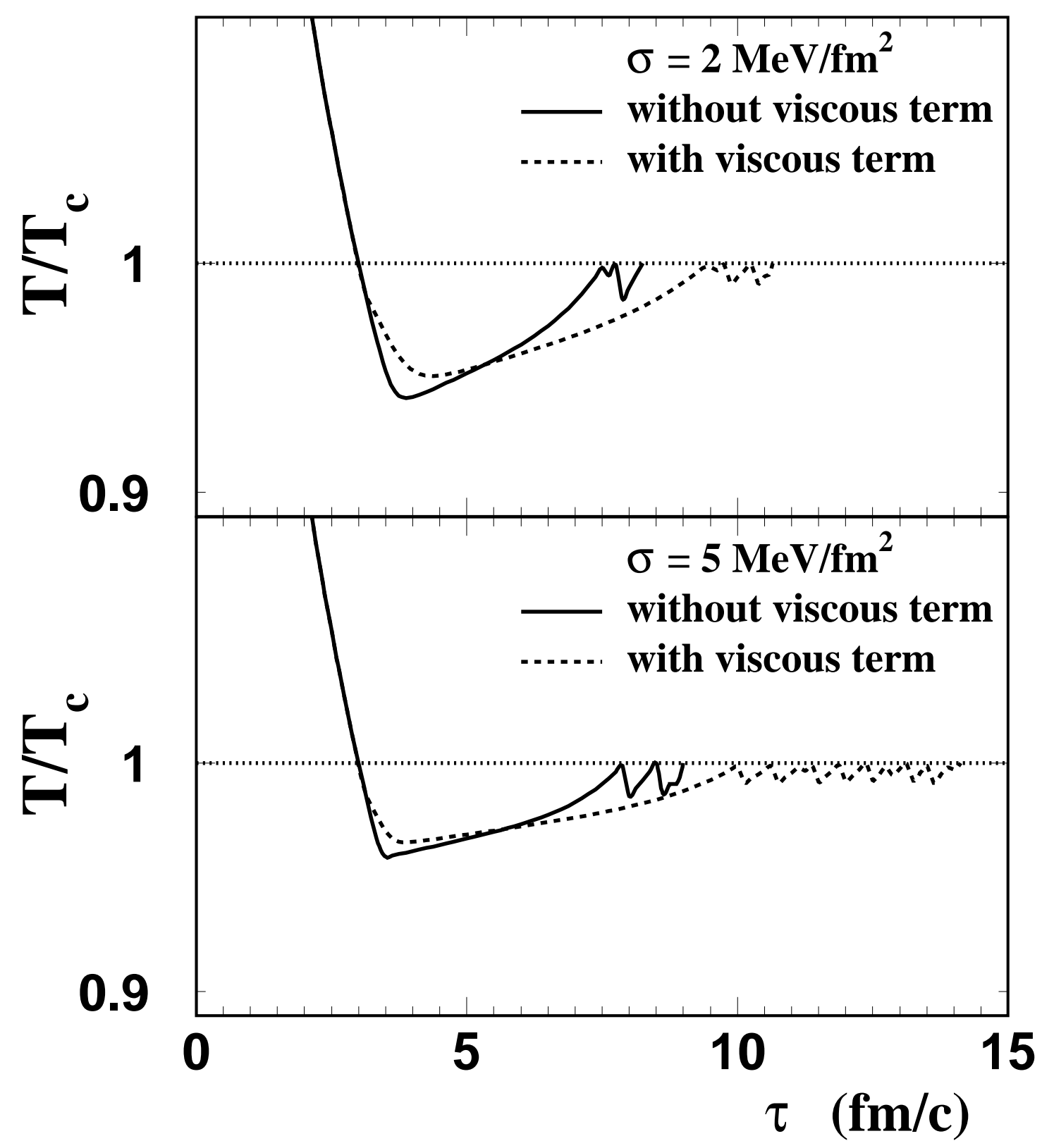

Fig. 2. The temperature as a function of the proper time for the phase transition QGP $\rightarrow$ hadrons during the longitudinal expansion. Upper and lower panel represents the results of calculations with $\sigma=2$ and $5 \mathrm{MeV} / \mathrm{fm}^{2}$, respectively. The solid/dashed curves correspond to calculations with Eq. (13)/Eq. (16). 


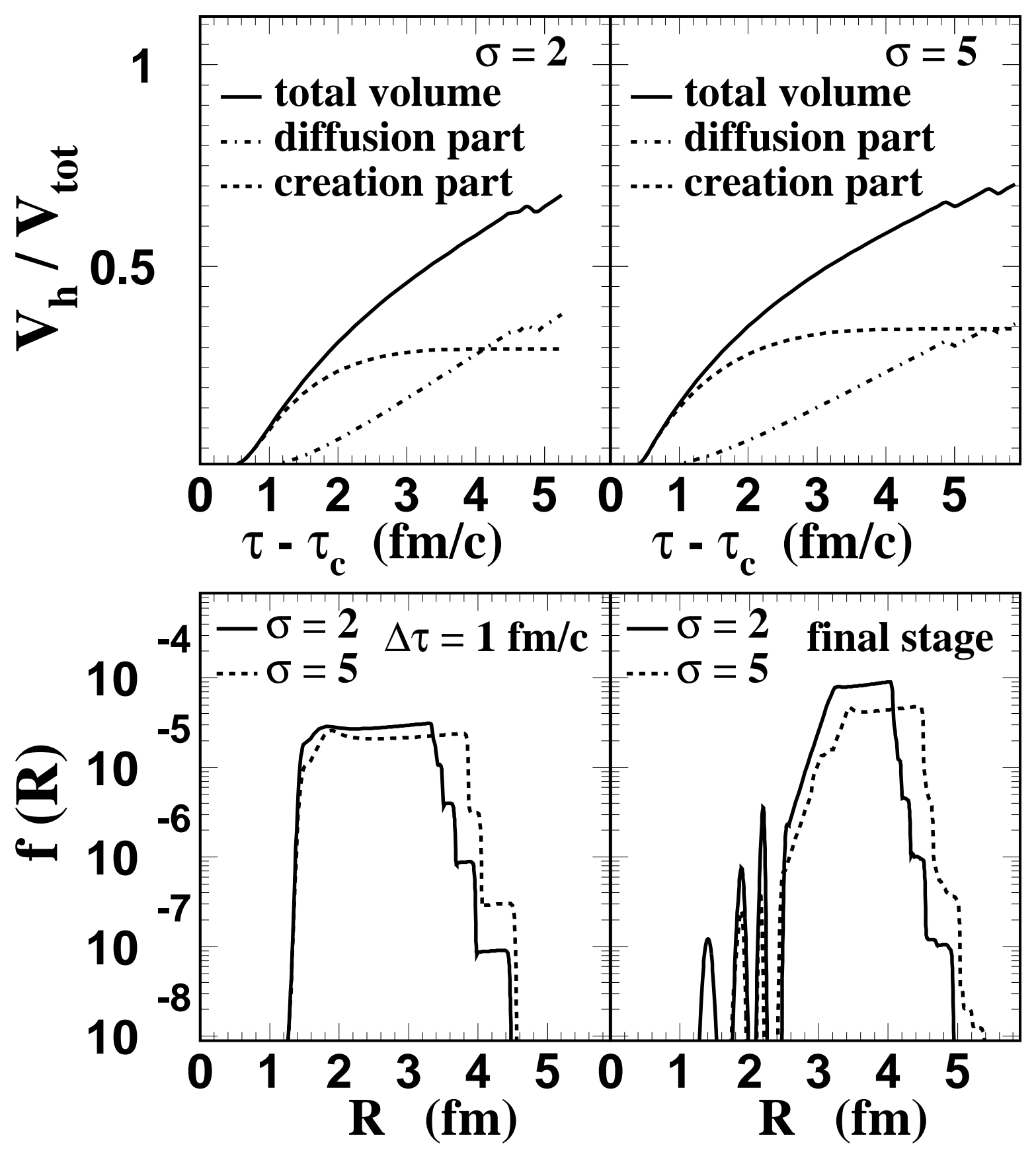

Fig. 3. Upper : The part of the QGP volume converted into hadrons for system with $\sigma=2$ (left panel) and $5 \mathrm{MeV} / \mathrm{fm}^{2}$ (right panel). Solid lines correspond to the total volume fractions of hadrons, dashed lines denote the increase of the hadronic volume due to the creation of new bubbles, and dash-dotted lines indicate the enlargement of the hadronic bubbles due to diffusion. Lower : Size distribution of the hadronic bubbles at $\Delta \tau=1 \mathrm{fm} / c$ after the beginning of the nucleation (left panel), and at the freeze-out (right panel) with $\sigma=2$ (solid curves) and $5 \mathrm{MeV} / \mathrm{fm}^{2}$ (dashed curves). 